\title{
Aplicação do índice prognóstico internacional em pacientes com linfoma difuso de grandes células $B$ em uma instituição brasileira
}

\author{
The use of the international prognostic index in a Brazilian institution for patients suffering from \\ diffuse large B-cell lymphoma
}

\author{
Abrahão E. Hallack Neto ${ }^{1,2}$ \\ Juliana Pereira ${ }^{2}$ \\ Pedro Dorlhiac-Llacer ${ }^{2}$ \\ Beatriz Beitler ${ }^{2}$ \\ Dalton A. F. Chamone ${ }^{2}$
}

\begin{abstract}
O linfoma difuso de grandes células B (LDGCB) corresponde a $50 \%$ dos casos de linfoma não-Hodgkin (LNH). A partir de 1993, o tratamento destes pacientes passou a ser direcionado pelo Índice Internacional de Prognóstico (IPI) validado em vários estudos. Entretanto a aplicação do IPI ainda não foi avaliada em nossa população e em nossas condições socioeconomicas. Neste estudo avaliamos o impacto do IPI ajustado para a idade (IPIa) na remissão completa (RC), sobrevida global (SG) e sobrevida livre de doença (SLD) dos portadores de LDGCB com idade inferior a 60 anos, tratados no Serviço de Hematologia do HCFMUSP. Dos 111 pacientes avaliados, 60 foram classificados com IPIa de risco baixo e intermediário e 51 IPIa de risco intermediário alto e alto. Os pacientes de risco baixo e intermediário foram analisados em conjunto no grupo de baixo risco adaptado e os de risco intermediário alto e alto no grupo de alto risco adaptado. Verificamos que a SG e a SLD foram influenciadas pelo estádio clínico, DHL e o estado funcional dos pacientes. Recomendamos o uso sistemático do IPI no tratamento dos nossos pacientes com LDGCB em nossa instituição. Rev. bras. hematol. hemoter. 2005;27(1):27-30.
\end{abstract}

Palavras-chave: Linfoma de grandes células; prognóstico; retrospectivo.

\section{Introdução}

Os linfomas não-Hodgkin (LNH) compreendem um grupo heterogêneo de neoplasias do tecido linfóide com distintos subtipos histológicos e apresentação clínica. Representam a quinta forma mais comum de câncer no Brasil, com incidência de 55 mil casos por ano e mais de 26 mil mortes. ${ }^{1}$ No município de São Paulo, a incidência em 1998 foi de 12,2/100 mil. ${ }^{2}$ Nos Estados Unidos, apresenta incidência de 8,5 casos $/ 100$ mil habitantes em indivíduos com idade inferior a 65 anos, e de 69 casos/100 mil habitantes acima de 65 anos. ${ }^{3}$ No Ocidente, o subtipo difuso de grandes células B (LDGCB) é o mais comum. ${ }^{4}$
Atualmente, as diretrizes terapêuticas baseiam-se no índice de prognóstico internacional (IPI), o qual pode ser adaptado para pacientes com idade inferior a 60 anos considerando-se apenas o estado funcional, a dosagem de DHL e o estádio. ${ }^{5}$ No Brasil, necessitamos de estudos que definam se o comportamento clínico e biológico dos pacientes com LDGCB é igual ou não ao descrito em outras áreas geográficas e se a terapêutica deve ser adaptada a estas novas condições.

Neste estudo avaliamos o IPIa dos pacientes com LGCB de 15 a 60 anos de idade tratados no Serviço de Hematologia do HC-FMUSP, verificando seu impacto nas taxas de RC, SG e SLD.

\footnotetext{
${ }^{1}$ Disciplina de Hematologia da Universidade Federal de Juiz de Fora.

${ }^{2}$ Disciplina de Hematologia e Hemoterapia da Faculdade de Medicina da Universidade de São Paulo.

Instituição em que foi realizado: Hospital das Clínicas da Faculdade de Medicina da Universidade de São Paulo.
}

Correspondência para: Abrahão E. Hallack Neto

Rua Oscar Vidal 90

36010-060 - Juiz de Fora-MG

Tel: 32-9112-6692

e-mail:abrahallack@ig.com.br 


\section{Casuística e Métodos}

Foram avaliados pacientes de 13 a 60 anos de idade com LDGCB diagnosticados entre janeiro de 1990 e dezembro de 2001 no Serviço de Hematologia do HC-FMUSP. Após autorização da comissão de ética foram avaliados prontuários médicos de forma retrospectiva. Foram excluídos pacientes com insuficiência cardíaca, hepática, renal, HIV positivos, falecidos nos primeiros trinta dias de tratamento, submetidos a transplante de medula óssea (TMO) e com prontuário médico incompleto. A data da biópsia foi considerada como data do diagnóstico e a última avaliação foi em dezembro de 2002. O estadiamento foi feito segundo os critérios de "Ann Arbor", considerando-se como "Bulky", massa tumoral superior a $7 \mathrm{~cm} .{ }^{6}$ Todos os pacientes foram tratados com esquemas contendo antraciclinas. Para cálculo da RC, SG e SLD, os pacientes de IPI de risco baixo e intermediário foram avaliados em um mesmo grupo denominado baixo risco adaptado, e os de IPI intermediário alto e alto no grupo de alto risco adaptado.

A SG foi analisada pelo método de Kaplan-Meier, considerando-se o período entre o início do tratamento e o óbito ou perda de seguimento (última avaliação) para a SG. A SLD foi calculada apenas para pacientes que obtiveram RC, a partir do diagnóstico até a data da progressão, recidiva, ou morte por qualquer causa. Os pacientes foram censurados na data do óbito ou abandono de tratamento. As curvas de sobrevida entre os diferentes grupos de risco foram comparadas pelo teste de long-rank. O impacto das diversas variáveis na sobrevida global foi verificado em análise multivariada pelo teste de regressão de Cox.

\section{Resultados}

\section{Características clínicas}

Foram avaliados 111 pacientes com idade média de 38 anos. Dos 111 pacientes, 71 (64\%) apresentavam sintomas B, $60,4 \%$ DHL acima do normal, 55\% "bulky", 43\% acometimento extranodal e $13 \%$ infiltração de medula óssea (MO).

Sessenta pacientes foram classificados em baixo risco adaptado, sendo vinte de baixo risco e quarenta de risco intermediário. Cinqüenta e um pacientes foram classificados como alto risco adaptado, sendo 33 de risco intermediário alto e 18 de alto risco (Tabela 1).

Oitenta e um $(73 \%)$ pacientes obtiveram RC (IC $(95 \%)=$ $64,7 ; 81,3)$ e 24 (27\%) foram resistentes primários. A SG, em sessenta meses, foi de $71 \%$, com média de 88 meses (IC $(95 \%)=$ $78,4 ; 97,8)$ (Figura 1). A SLD foi de 59\% em sessenta meses com média de 93,3 meses (IC(95\%)=73,9; 112,8) (Figura 2). Vinte e seis (32\%) recidivaram, a maioria dos quais, de risco intermediário alto e alto. (Tabela 2).

Fatores de prognóstico

A RC dos pacientes com estádios I e II foi de $83,1 \%$, a

\begin{tabular}{ccc}
\multicolumn{3}{c}{ Tabela 1 } \\
Características clínicas \\
\hline & $\mathrm{n}$ & Total \\
\hline F & 53 & 47,7 \\
M & 58 & 52,3 \\
Idade (anos) & 38 & - \\
Sintomas B & 71 & 64 \\
Extranodal & 48 & 43,2 \\
MO+ & 14 & 12,6 \\
"Bulky" & 61 & 55 \\
I e II & 59 & 53,2 \\
III e N & 52 & 46,8 \\
Baixo risco adaptado & 60 & 54,1 \\
Alto risco adaptado & 51 & 45,9 \\
\hline Total & 111 & 100
\end{tabular}

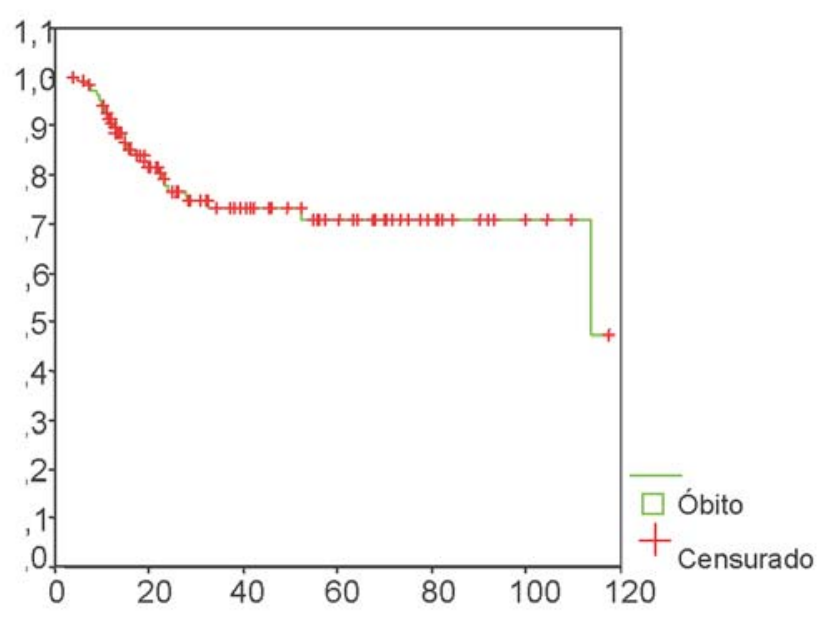

SG - Meses

Figura 1. Curva de Sobrevida Global

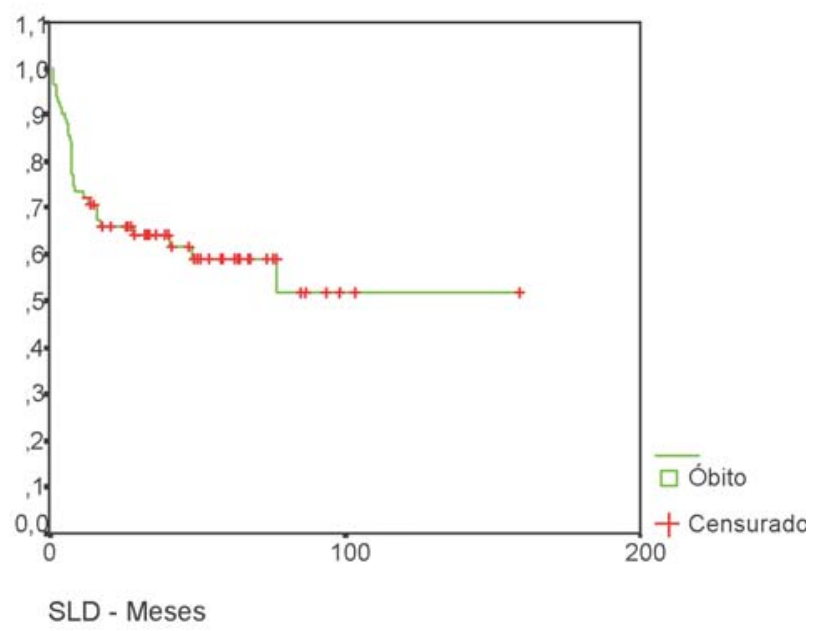

Figura 2. Curva de Sobrevida Livre de Doença 


\begin{tabular}{ccc} 
Tabela 2 & \multicolumn{2}{c}{ Total } \\
Características dos pacientes refratários e recidivados \\
\hline & \multicolumn{3}{c}{ N } & $\%$ \\
\hline DHL > normal & 34 & 68 \\
I e II & 17 & 34 \\
III e IN & 33 & 66 \\
Sintomas B & 40 & 80 \\
Extranodal & 21 & 42 \\
"Bulky" & 28 & 56 \\
MO+ & 10 & 20 \\
Baixo risco adaptado & 21 & 42 \\
Alto risco adaptado & 29 & 58 \\
\hline Total & 50 & 100
\end{tabular}

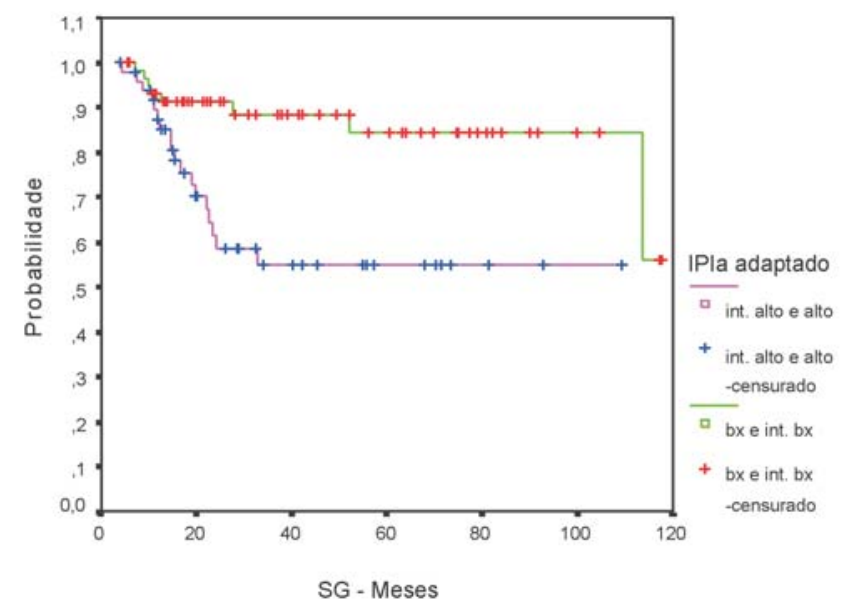

$p=0,002$

Figura 3. SG com relação ao IPla

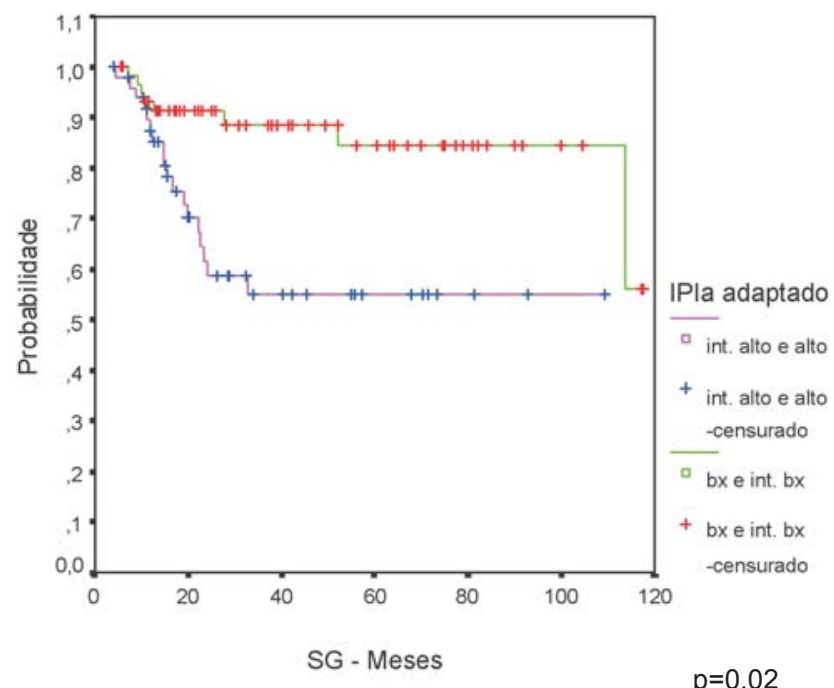

Figura 4. SLD com relação ao IPla

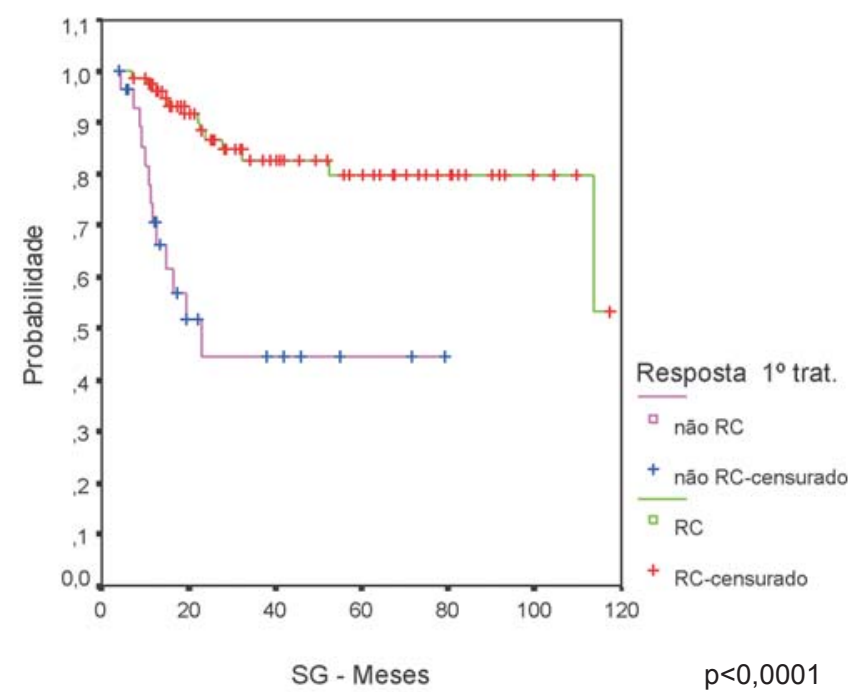

Figura 5. SG com relação ao RESP1CAT

SG de $84,2 \%(\operatorname{IC~}(95 \%)=90,8 ; 11,8)$ e a SLD de $74,8 \%$ em sessenta meses $(\mathrm{IC}(95 \%)=62,6 ; 86,8)$. A RC dos 52 pacientes com estádios III e IV foi de $61,5 \%$ e a SG e a SLD em sessenta meses de 55,1\% e 37,1\%, respectivamente. A média de SG foi de 73,4 meses (IC $(95 \%)=57,7 ; 89,1)$ e de SLD de 65,7 meses $(\mathrm{IC}(95 \%)=39,57 ; 91,88)$. As taxas de RC, SG e SLD dos pacientes com estádios I e II foram estatisticamente superiores aos daqueles com estádios III e IV ( $p=0,011,0,0124$ e 0,0004). Em análise univariada, DHL e MO infiltrada tiveram impacto negativo nas SG e SLD ( $p=0,009$ e 0,011).

No grupo baixo risco adaptado, a RC foi de $78,3 \%$, a SG de $84,3 \%($ IC $(95 \%)=91,7 ; 112,1)$ e a SLD de $68,12 \%$ em sessenta meses $(\mathrm{IC}(95 \%)=56,7 ; 82,5)$. No grupo alto risco adaptado, a RC foi de 66,7\%, a SG de 54,8\% (IC (95\%) = 53,1; $82,5)$ e a SLD de $46,6 \%$ em sessenta meses $(\operatorname{IC~}(95 \%)=53,5$; $105,7)$, com significância estatística para a SG e SLD ( $p=$ 0,002 e 0,02 ) (Figuras 3 e 4 ). Os pacientes em RC com o primeiro tratamento apresentaram SG em sessenta meses de 79,7\% $(\mathrm{IC}(95 \%)=87,9 ; 107,4)$, estatisticamente superior à dos resistentes primários ( $\mathrm{p}<0,00011)$ (Figura 5).

Quando realizada a análise multivariada com os fatores que influenciaram a SG, mostraram-se como fator preditor independente o IPIa $(p=0,009)$ com risco relativo $(\mathrm{RR})$ de 3,2 $(\mathrm{IC}(95 \%)=1,33 ; 7,75)$ e a resposta a terapêutica primária $(\mathrm{p}<0,0001)$ com RR de 4,61 (IC $(95 \%)=2,07 ; 10,3)$.

\section{Discussão}

Em nossa casuística, as taxas de RC, SG e SLD foram semelhantes às da literatura. Entretanto, houve maior incidência de pacientes com sintomas B e "Bulky", o que pode estar associado à dificuldade de acesso ao serviço público de saúde. Nossa padronização de "Bulky" como massa superior a $7 \mathrm{~cm}$ pode ter colaborado para sua maior incidência em nossa população. A presença de "Bulky" foi um fator de 
mau prognóstico para a obtenção de $\mathrm{RC}$, mas não para as SG e SLD7.

O fato de que não haver diferença nas SG e SLD entre os pacientes de IPIa de risco intermediário baixo e baixo, e entre pacientes de IPIa intermediário alto e alto risco, nos permitiu remanejar os pacientes para os grupos de baixo risco adaptado e alto risco adaptado. ${ }^{8}$ Os pacientes do grupo de baixo risco adaptado apresentaram SG e SLD estatisticamente superiores aos dos pacientes de alto risco adaptado. A variável com maior impacto para a SG foi a obtenção de $\mathrm{RC}$ com o primeiro tratamento ( $\mathrm{p}<0,0001)$. Dos 81 pacientes que obtiveram RC, 47 (58\%) eram de baixo risco adaptado, e na análise mulivariada esses dois fatores foram preditores independentes para SG.

Assim como no estudo em que se padronizou o IPI em pacientes com idade inferior a 60 anos, a presença de doença extranodal não influenciou a SG ou a SLD em nossa casuística. ${ }^{5}$ Entretanto, recentemente, Moller realizou estudo com grande número de pacientes portadores de LDGCB e verificou que o acometimento extranodal teve impacto negativo na sobrevida dos pacientes, independentemente da idade. ${ }^{9}$

Assim como na literatura, a infiltração de MO e DHL acima do normal foram fatores de mau prognóstico para a SLD e SG, respectivamente. Verificamos também que o aumento de DHL foi fator de mau prognóstico independente dos outros parâmetros do IPIa. ${ }^{5,10,11}$

Confirmamos que, também em nossa população e em nossas condições socioeconômicas, o IPIa é de extrema importância na condução terapêutica dos pacientes com LDGCB. Sugerimos a aplicação sistemática do IPI na estratificação de risco dos pacientes com LDGCB em nossa população.

\section{Abstract}

Diffuse Large B-Cell Lymphomas (DLBCL) correspond to $50 \%$ of non-Hodgkin's lymphomas. Since 1993 the treatment of these patients has been directed by the International Prognostic Index (IPI), validated in several studies. However, the use of the IPI has not been evaluated in our population and social-economical conditions. In this study, we evaluate the impact of the age-adapted IPI (aIPI) in the complete response, overall survival and disease-free survival in under 60-year-old DLBCL sufferers treated in the Hematology Service of HCFMUSP. Of the 111 evaluated patients, 60 were classified as aIPI low and intermediate risk and 51 as aIPI intermediate-high and high risk. The patients with low and intermediate risk were analyzed as a whole with adapted low risk and patients with intermediate-high and high risk with the adapted high risk. We verified that the overall survival and disease-free survival were influenced by the clinic stage, $L D H$ value and patients performance status. We recommend the regular use of IPI in the treatment of the patients with DLBCL in our institution. Rev. bras. hematol. hemoter. $2005 ; 27(1): 27-30$.

Key words: Large cell lymphoma; prognosis; retrospective.

\section{Referências Bibliográficas}

1. Bigni R. Inst. Nac. Câncer. Linfoma não Hodgkin, citado em: 29 junho 2004. Disponível em: http://www.gov.br

2. Mirra AP, Latorre MRDO, Veneziano DR, eds. Incidência de câncer no município de São Paulo, Brasil: 1997-1998. Mortalidade de câncer no município de São Paulo, Brasil, tendência no período 1969-1998. São Paulo, Ministério da Saúde; 2001.

3. Rabkin CS, Ward MH, Manns A et al. Epidemiology of nonHodgkin's lymphomas. In: Magrath IT. The Non-Hodgkin's Lymphomas. London, Arnold;1997; p.171-86.

4. Shih LY, Liang DC. Non-Hodgkin's Lymphomas in Asia. Hematol Oncol Clins N America 1991;5:983-1001.

5.The International non-Hodgkin's lymphoma prognostic factors project. A predictive model for aggressive non-Hodgkin's lymphoma. New Engl J Med 1993;329: 987-94.

6. Carbone PP, Kaplan HS, Musshof K et al. Report of the Committee on Hodgkin's Disease Staging Classification. Cancer Res 1971;31: $1860-1$.

7. Shipp MA, Mauch PM, Harris NL. Non-Hodgkin's Lymphomas. In: De Vita Jr VT, Hellmans S e Rosenberg SA, eds. Cancer principles and practice of oncology. 5.ed. Philadelphia, Lippincott-Raven, 1997.

8. Gordon LI, Andersen J, Colgan J et al. Advanced diffuse nonHodgkin's lymphoma: Analysis of prognostic factors by the International Index and by lactic dehydrogenase in an intergroup study. Cancer 1995;75:865-73.

9. Moller MB, Pedersen NT, Christensen BE. Diffuse large B-cell lymphoma: clinical implications of extranodal versus nodal presentation-a population-based study of 1575 cases. Br J Haematol 2004;124(2):151-9.

10. Nicolaides C, Fountzilas G, Zoumbos N et al. Diffuse large-cell lymphomas: Identification of prognostic factors and validation of the International Non-Hodgkin's Lymphoma Prognostic Index. A Hellenic Cooperative Oncology Group Study. Oncology 1998; 55:405-15.

11. Milpied N, Deconinck E, Gaillard F et al. Initial treatment of aggressive lymphoma with high-dose chemotherapy and autologous stem-cell support. N Engl J Med 2004;350:1.287-95.

Avaliação:

Carlos S. Chiattone, editor associado, e mais dois revisores externos. Publicado após concordância do editor.

Conflito de interesse: não declarado

Recebido: 14/09/04

Aceito após modificações: 07/03/05 